\title{
NUMERICAL ANALYSIS OF A FORGING PROCESS FOR PRODUCING A HOLLOW BALL FROM TUBE
}

\author{
Grzegorz Samołyk ${ }^{1}$ \\ 1 Department of Computer Modelling and Metal Forming Technologies, Mechanical Engineering Faculty, \\ Lublin University of Technology, Nadbystrzycka 36, 20-618 Lublin, Poland, e-mail: g.samolyk@pollub.pl
}

Received: 2018.06.11

Accepted: 2018.08.01

Published: 2018.09.01

\begin{abstract}
The paper presents the results of a numerical analysis (by FEM) of a new forging process for producing a hollow ball with an outside diameter of $30 \mathrm{~mm}$ and a wall thickness of $4.4 \mathrm{~mm}$. It was assumed that the ball forging would be cold formed from hollow billet (tube) made of $19 \mathrm{MnCr} 5$ steel. The effect of billet dimensions (diameter, height and wall thickness) on the forming process was investigated, which led to identification of basic shape defects and determination of the billet dimensions required for producing balls of the desired geometry. The ball forming conditions, including force parameters of the process, are thoroughly investigated.
\end{abstract}

Keywords: hollow ball, die forging, cold forming, $19 \mathrm{MnCr} 5$ steel.

\section{INTRODUCTION}

Balls are widely used as elements of many parts of machines and devices, and their application is universal. These elements can be made of various materials, as solid or hollow products with a wide range of diameters. For example, they are widely used in rolling bearings, ball valves and industrial mills. Elements of this type are produced using various techniques - depending on the need, these are casting, forging or rolling methods and, in some cases, even machining methods. However, in industrial conditions, metal forming-based methods are predominantly used because they enable the shaping of desired mechanical properties of final products and are economically justified, particularly in large-lot or mass production.

Extensive research on new rolling processes for balls (of general purpose or as elements for ball mills) was carried out by Pater and Tomczak, and the obtained results were described, for example, in [1]. The authors of this work showed that balls with diameters bigger than 40 $\mathrm{mm}$ can be effectively formed by helical rolling
[2], wherein the billet is made of fragments of a scrap railway rail. An equally effective process is cross-wedge rolling [3], which can also be used for the production of balls with diameters smaller than $40 \mathrm{~mm}$. An equally interesting technological solution is the multi-operation forming of solid balls by the forging method, and then calibrating them by rolling [4].

Hollow balls are predominantly produced by methods developed for the manufacturing of semi-finished products, which are then used to produce elements for ball valves. A characteristic of such products is a cylindrical through-hole with a relatively large diameter in comparison to the outside diameter (of the ball). Such forgings can be produced by rolling on mandrel [5], e.g. using a rolling mill for manufacturing rings, or forged using specially designed dies $[6,7,8]$. For instance, in single-operation forming of a forging [7] but with a blind hole (an additional operation of web cutting is required [8]) or two-operation mandrel forging [6].

Forgings of hollow balls characterized by a relatively constant wall thickness can be formed from hollow billet by the rotary com- 
pression method [9] or die forging [10, 11]. However, the well-known methods have their limitations. For example, rotary compression [9] is performed under hot forming conditions, and produced ball forgings are connected and must be cut. On the other hand, in the forging process by the Eklund and Campbell method [10], forgings of balls are produced in two or three forming operations, using dies that additionally rotate around the main axis. This, in turn, makes the industrial application of this method requires the use of specially designed forging machines.

A survey of the state of the art in the field of ball-forming techniques allowed for proposing a new forging method for producing hollow forgings. The forging process is performed in cold forming conditions, in one operation, with closed dies and a universal forging machine, e.g. a hydraulic press. In a further part of this paper, the above-mentioned method will be described in detail, and selected results of the numerical simulation will be reported and discussed.

\section{DESIGN OF THE PROPOSED DIE FORGING PROCESS}

A general design of the proposed die forging process for a hollow ball is shown schematically in Fig. 1. It is assumed that this process would be performed on a hydraulic press under cold forming conditions in order to ensure higher properties of the forging than could be obtained by a hot forging process.
In this forging process, a hollow billet 1 is placed in a lower die impression 5 with the use of a feeding and centring device 6 . This device can be an arm grip of an industrial manipulator, which will ensure precise and reproducible position of the billet. Next, an upper die 4 performs working motion with a constant velocity $v_{l}$, which, in effect, begins the formation of the workpiece, and at the appropriate moment, the feeding and centring device 6 is retracted from the working space at a relevant speed $v_{2}$. The end result is a forging of ball 2 with a variable wall thickness $g_{K}$ and two technological holes 3 with a resultant diameter $d_{K}$.

The produced forging of a hollow ball is a semi-finished product with a wide range of applications. For example, according to the concept of Eklund and Campbell [10], technological holes can be made "blind" and the end result is a full-value ball with a lower mass than that of a solid ball. Since the forging was produced by cold forming, this enables a significant reduction in the labour consumption of successive finish machining.

The produced ball forging can also be used as a semi-finished product for making a ball valve. In this case, the inner hole with a constant diameter can be obtained, for example, by forcing in a thin-walled sleeve. This technological solution was used, for example, by Chiuchang [11] for the production of ball valves made of thin sheets by two-roll compression.

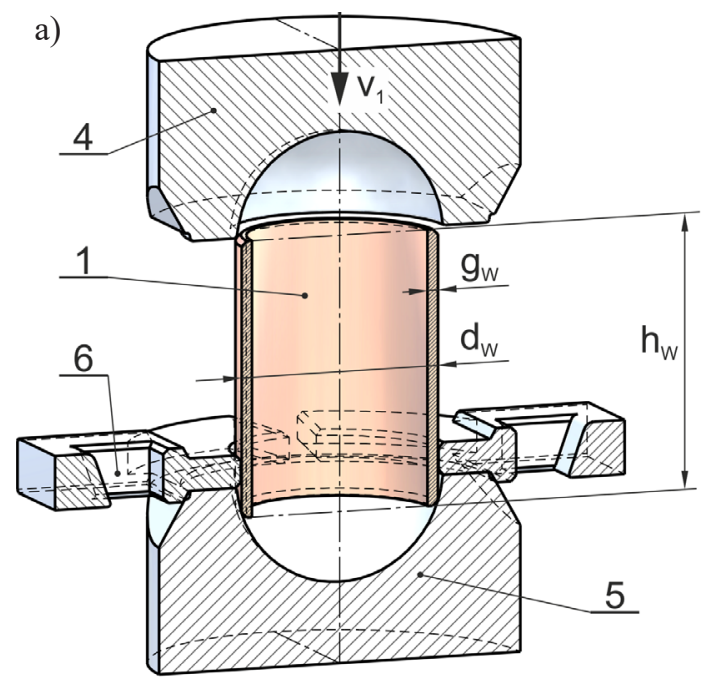

b)

Fig. 1. Schematic design of the die forging process for a hollow ball: a) beginning of the process, b) final stage of the process; description in the text 


\section{DESCRIPTION OF THE CONDITIONS APPLIED IN THE SIMULATION OF A FORGING PROCESS FOR A HOLLOW BALL}

The object of the numerical analysis (performed by the finite element method) is a die forging process for producing a hollow ball, schematically shown in Fig. 1. It is intended to obtain a ball with the outside diameter of $D_{K}=30 \mathrm{~mm}$, where the arbitrary wall thickness is equal to $g_{N}=$ $4.40 \mathrm{~mm}$. The design task boils down to ensuring stable forming conditions of the forging process and obtaining technological holes with the smallest possible diameter $d_{K}$.

It should be clarified that the proposed method does not make it possible to produce a hollow ball with constant wall thickness. According to the diagram shown in Fig. 1b, the minimum wall thickness $g_{K}$ will differ from the arbitrary thickness $g_{N}$, and the maximum thickness $g_{M A X}$ will occur in the vicinity of the technological hole with a diameter $d_{K}$ - this problem will be discussed in more detail later on in this paper. Therefore, the previously given thickness $g_{N}$ is a theoretical (calculation-related) parameter proposed by the author, assuming that the hollow ball has a constant wall thickness and no technological holes are formed. This enables the determination of the forging volume and, on this basis, the determination of required dimensions of the hollow billet (Fig. 1a). Therefore, the numerical analysis was performed for the cases of forging a hollow ball from a hollow billet with the wall thickness $g_{W}$ of $3.60,3.80$ and $4.00 \mathrm{~mm}$, respectively. Then, by selecting the outside diameter $d_{W}$ from the range of $23.5 \mathrm{~mm}$ to $27.5 \mathrm{~mm}$, a corresponding billet height $h_{W}$ was determined. Obtained heights were in the range from $32.5 \mathrm{~mm}$ to $40.9 \mathrm{~mm}$, which causes that the slenderness ratio $h_{W} / d_{W}$ is between $1.18 \mathrm{~mm}$ and $1.74 \mathrm{~mm}$, while the $d_{w} / g_{w}$ ratio ranges from $5.88 \mathrm{~mm}$ to $7.36 \mathrm{~mm}$. According to Alhussain's research [12], the upsetting of hollow billet at these values of the slenderness ratio can only pose a risk of local buckling, which - according to the author - should not be a greater obstacle in maintaining stable conditions of the forming process for a ball. A list of cases subjected to numerical analysis is presented in Table 1 .

The analysed process was numerically modelled with the use of the commercial simulation programme Deform-3D, based on the finite element method (FEM). Its usefulness for numerical modelling of metal forming processes has been repeatedly confirmed by experimental verification, as described, for example, in $[1,3,13]$. In the calculations, it was assumed that the forming process would be performed under cold forming conditions (the initial temperature of the billet and the tools was set to $\mathrm{T}=20^{\circ} \mathrm{C}$ ); however, the simulation took account of thermal phenomena closely related to the work of plastic deformation and friction. It was assumed that the heat exchange coefficient between the workpiece material and the tools was $10 \mathrm{~kW} / \mathrm{m}^{2} \mathrm{~K}$. The phenomenon of friction was described with a constant friction model, assuming that the friction factor between the workpiece and the tools is $\mathrm{m}=0.15$, which corresponds to good lubrication conditions. The condition of mutual contact between the surfaces of the billet is also met, assuming that $\mathrm{m}=0.5$.

The simulation assumed that the forging would be forged on a hydraulic press ensuring a constant velocity of motion of the upper die at $v_{1}$ $=40 \mathrm{~mm} / \mathrm{s}$. For obvious reasons, the aspects of the feeding and alignment device were omitted. In addition, it was assumed that the forging would be made of $19 \mathrm{MnCr} 5$ alloy steel in the softened state.

Table 1. Dimensions of the hollow billet (denoted in accordance with Fig. 1a) and IDs of cases analysed by FEM

\begin{tabular}{|c|c|c|c|c|c|c|}
\hline \multirow{2}{*}{$\begin{array}{l}\text { Diameter } d_{w} \\
{[\mathrm{~mm}]}\end{array}$} & \multicolumn{2}{|c|}{ Wall thickness $\mathrm{g}_{\mathrm{w}}=3.6 \mathrm{~mm}$} & \multicolumn{2}{|c|}{ Wall thickness $\mathrm{g}_{\mathrm{w}}=3.8 \mathrm{~mm}$} & \multicolumn{2}{|c|}{ Wall thickness $g_{w}=4.0 \mathrm{~mm}$} \\
\hline & ID of case & $\begin{array}{l}\text { Height } h_{w} \\
{[\mathrm{~mm}]}\end{array}$ & ID of case & $\begin{array}{l}\text { Height } h_{w} \\
{[\mathrm{~mm}]}\end{array}$ & ID of case & $\begin{array}{l}\text { Height } h_{w} \\
{[\mathrm{~mm}]}\end{array}$ \\
\hline 23.5 & $\mathrm{C} 1$ & 40.9 & - & - & $\mathrm{D} 1$ & 37.6 \\
\hline 24.0 & $\mathrm{C} 2$ & 39.9 & B2 & 38.2 & - & - \\
\hline 24.5 & $\mathrm{C} 3$ & 39.9 & B3 & 37.3 & D3 & 35.8 \\
\hline 25.0 & $\mathrm{C} 4$ & 38.0 & B4 & 36.4 & D4 & 34.9 \\
\hline 25.5 & C5 & 37.2 & B5 & 35,5 & D5 & 34.1 \\
\hline 26.0 & C6 & 36.3 & - & - & - & - \\
\hline 26.5 & $\mathrm{C} 7$ & 35.5 & B7 & 34.0 & D7 & 32.6 \\
\hline 27.5 & - & - & B8 & 32.5 & - & - \\
\hline
\end{tabular}


The material model of this steel can be described with the constitutive equation of the form [14]:

$\sigma_{\mathrm{p}}=1398 \cdot \varphi^{0.192} \cdot \exp (-0.41 \varphi) \cdot \varphi^{\left(0.01+8 \cdot 10^{-5} \cdot \mathrm{T}\right)} \cdot \exp (-0.0017 \cdot \mathrm{T})(1)$ where: $\sigma_{\mathrm{p}}$ - yield stress, $\varphi$ - effective strain, $\dot{\varphi}^{-}-$ strain rate, $\mathrm{T}-$ absolute temperature of the forging.

In order to minimize the calculation time and to increase the accuracy of obtained results, the simulation considered a three-dimensional case of forming a small section of the forging ( $1 / 4$ of the perimeter), which was divided into about 150,000 tetragonal elements. The boundary conditions of symmetry were defined on the edges of this section. The possibility of using a $1 / 8$ section of the ball in the calculations was also considered; however, the preliminary calculations showed that due to a number of phenomena disturbing the stability of the forming process, such a far-reaching simplification is not recommended. Also, for the same reason, a simulation variant involving the use of an axi-symmetric two-dimensional model was rejected.

\section{GENERAL DESCRIPTION OF THE FORGING PROCESS}

The forging process for producing hollow balls from hollow billet consists in forming the workpiece by upsetting and compressing it in spherical impressions at the same time. This means a complex patter of metal flow. In addition, given the relationships between individual dimensions of the billet, the stability of the forging process can easily be interrupted, which leads to the occurrence of certain shape defects of the forging. Fig. 2. shows a diagram in the coordi- nates of two ratios defines based on the billet dimensions (Fig. 1a). Points marked in the diagram are an arbitrary representation of numerical results of the simulated cases listed in Tab. 1. The results were classified based on the geometric defects of the forging, as additionally shown in Fig. 3 for three representative cases.

The process of forging hollowed-out balls from tubular batch is reduced to shaping the material by simultaneous upsetting and crimping in a spherical cut. This is a complex flow diagram of the material. Considering the additional interdependencies between the dimensions of the batch, the stable course of the forging process can easily be disturbed, which in turn leads to specific errors in the shape of the forging. Fig. 2. shows a diagram for the coordinates of two ratios defined on the basis of batch dimensions (Fig. 1a). The points on the diagram represent the simulated numerical simulation results of the cases listed in Table 1. The result is classified based on geometrical defects of the forging, which are additionally shown in Fig. 3 for three representative cases.

As it can be seen in Fig. 2, there is a very narrow range of billet dimensions ensuring the production of a forging with correct shape. The numerical results reveal that the mutual ratio of the billet dimensions is of key importance, and standard shape defects occur not only with relation to the outer profile of the workpiece (dominant and always taken into account) but can also pertain to the internal outline of the workpiece. If the $h_{w}$ ' $d_{w}$ ratio is too high (above 1.5 approx.), the material undergoes buckling due to underfill resulting from the formation of workpiece surface concavity (Fig. 3a, Detail A). The higher the value of $d_{w} /$

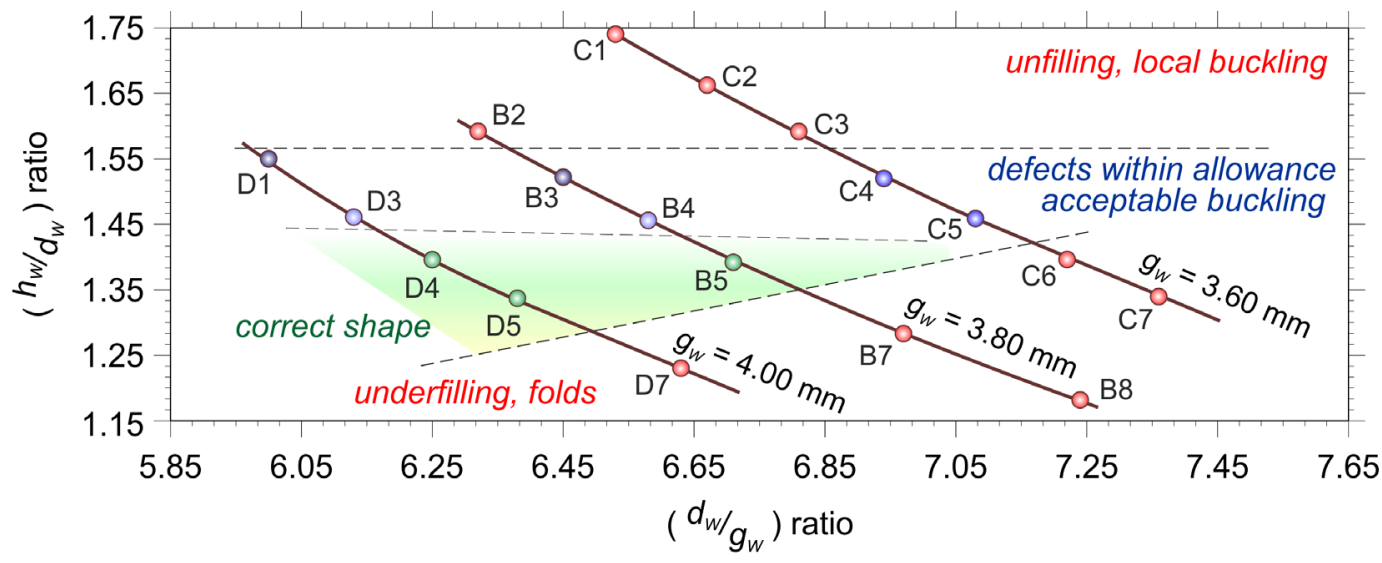

Fig. 2. Characteristic of the numerical results with respect to potential shape defects of the workpiece; denotations according to with Fig. 1a and Table 1 

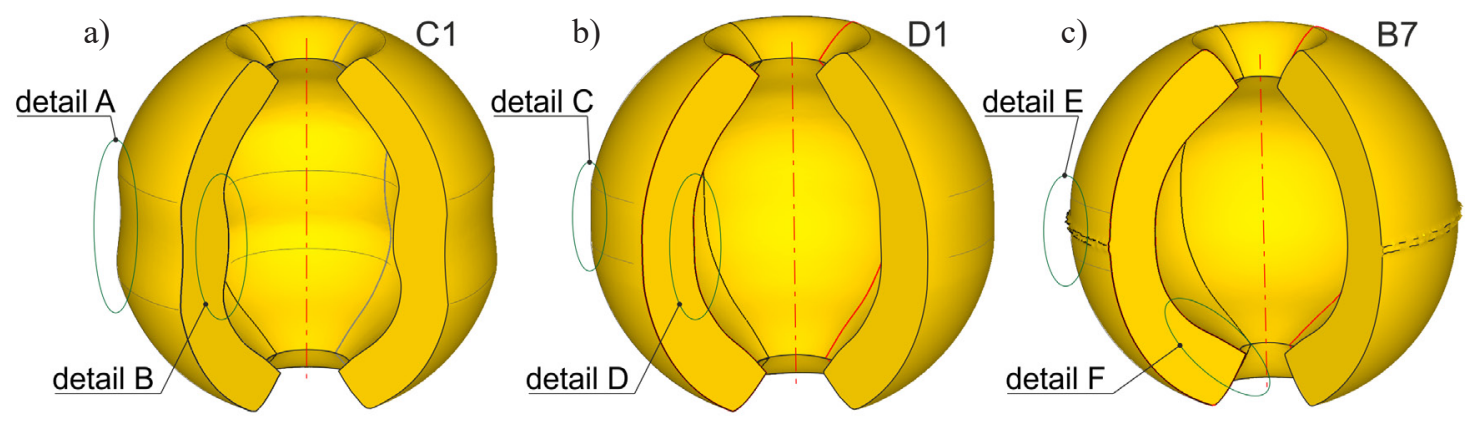

Fig. 3. Examples of typical shape defects of the ball; description in the test

$g_{w}$ is, the more considerable this defect becomes (and the less stable the forming process is). When the hw/dw ratio reaches its limit value, the buckling disappears, while the minimal tendency for stability loss can only lead to the formation of small flattening of the ball surface in the vicinity of the die parting plane (Fig. 3b, Detail C). This defect can also occur when the billet diameter $d_{W}$ is insufficiently smaller than the ball diameter $d_{K}$. This flattening is associated with underfill and can be regarded, for instance, as the so-called defect lying in the tolerance range of machining allowance. On the other hand, the defects of the internal surface of the forging (Fig. 3, Details B and D) result from the formation of external defects mentioned above.

The use a billet with a smaller $h_{w} / d_{w}$ ratio can lead to overfill during the forging process, which leads to the formation of undesired flash in the die parting plane. Before the dies close, one can observe the "cutting" of the workpiece surface by the edges of the closing die impression, which leads to fold (Fig. 3c, Detail E). This defect is unacceptable. It should be explained that this situation results from selecting a too high value of the diameter $d_{W}$ relative to the ball diameter $d_{K}$, while during upsetting the wall thickness $g_{W}$ is so high that there occurs radial flow of the metal outwards. All this leads to the excessive increase in the wall thickness of the forging in the vicinity of the technological hole (Fig. 3c, Detail F). It should be added that the arbitrary diameter $d_{K}$ of the technological hole (Fig. 1b) is a resultant parameter, and its arbitrary selection is not an easy task due to the presence of a number of failure modes affecting the forming process stability.

\section{DETAILED DESCRIPTION OF THE FORMING PROCESS}

According to Fig. 2, hollow balls with correct geometry can only be produced if hollow billet with specified dimensions is used. In this investigation, the desired results were obtained only in three cases (according to Table 1 they were: B5, D4, D5). An analysis of the results demonstrates that the billet diameter $d_{W}$ should be proportionately smaller than the ball diameter $d_{K}$ and, at the same time, the wall thickness $g_{W}$ cannot be too small relative to either the theoretical thickness

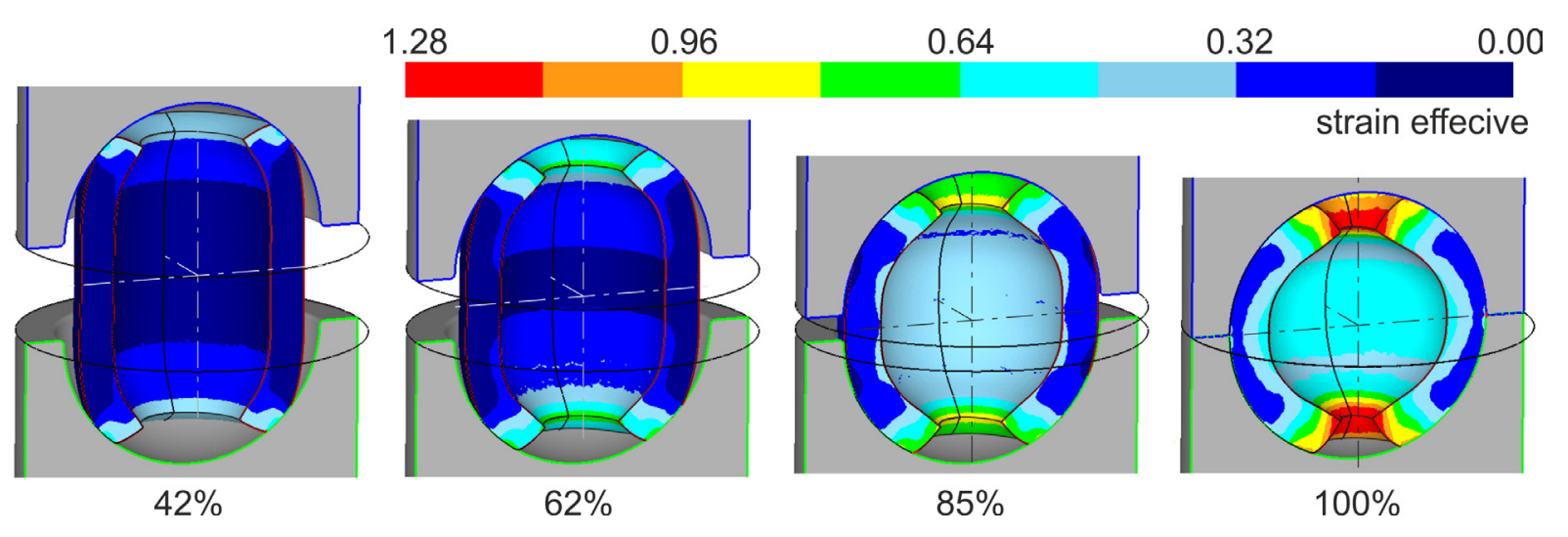

Fig. 4. Changes in the shape and effective strain during the forming of ball from billet B5 
value $g_{N}$ or other dimensions of the billet. Fig. 4 shows selected stages of the forging process for a ball made of billet B5; it can be observed that initially the billet undergoes significant reduction, which leads to the formation of a technological hole. It is not until later (process advancement over $40 \%$ ) that the billet undergoes clear upsetting, which is caused by an increase in the contact surface between the material and the impression (more precisely, the increase in the friction force in the vicinity of the hole being formed). In a further stage of the ball formation one can primarily observe an increase in the billet diameter in the die parting plane and a slower decrease in the hole dimeter in the upper part of the forging, with a simultaneous rapid increase in the wall thickness of the ball in the vicinity of the this hole . This is also confirmed by the calculated distribution of effective strains, as shown in Fig. 4. This distribution also proves that the pattern of metal flow is to a certain extent similar to the pattern of bending along two lines that are symmetrically distant from the die parting plane. This is also confirmed by the way of stability loss of the workpiece shape in the case shown in Fig. 3a.

Fig. 5 illustrates the variations in the forming force observed for three cases of the forging process for balls. Based on the diagram, the forming process can be divided into two stages. The first stage is characterized by a monotonous increase in the force, which means the process is steady and stable. There is only a rapid increase in the force value at the beginning of this stage, which can be connected with the moving of the workpiece end over the die impression surface and may indicate that the billet must be maintained in position by the feeding and aligning device (Fig. 1, Component 6), but only until the force becomes stable.

The stage of a stable increase in the force ends when the die impression is filled with material (Fig. 5, Detail A) and the calibration of the ball external surface begins. In the diagram this moment is marked as a rapid increase in the force. This is typical of closed die forging processes, however - when compared to the forging of solid parts $\neg$ the maximum forging force for producing a hollow ball is relatively low. The maximum forces calculated for the three analysed cases are shown in Fig. 5. Interestingly, for B5 (i.e. billet with a lower thickness $g_{W}$ ), the force is lower for the longer part of the forging process than in other cases, but its maximum value if higher (fortunately, the difference in values of $F_{\max }$ is insig-

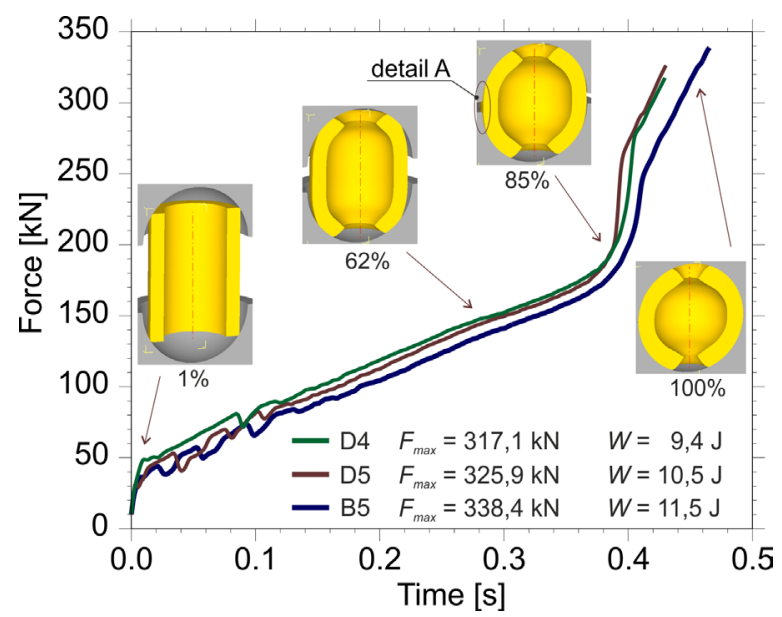

Fig. 5. Comparison of the ball forming force and the unit work $W$ performed by the tool for three forging cases

nificant). On the other hand, the longer forming path (due to a higher initial height $h_{W}$ ) leads to a clear increase in power demand.

Fig. 6 shows the cross section of the forged ball, along with the distribution of the wall thickness $g_{K}$ (measured every $15^{\circ}$ ), the dimensions of the technological hole $d_{K}$ and the ball height measured over the principal (vertical) axis going through the above-mentioned holes. The ball diameter $\mathrm{D}$ is constant as expected and amounts to $30 \mathrm{~mm}$. The figure shows results for three cases. The produced hollow balls are characterized by a varying wall thickness and irregular shapes of the technological holes (quasi-conical surface). The highest wall thickness is observed in the vicinity of the above holes, however in the closest proximity to the hole - this wall thickness (measured along the straight line crossing through the centre of the ball) decreases to zero. Much lower values of $g_{K}$ that are, the same time, closer to the standard value $g_{N}$, occur in the neighbourhood of the die parting plane. The wall thickness measured only on the die parting plane is slightly higher, which is caused by the upsetting of material in this zone, in the final stage of the process.

It was also observed that the wall thickness is higher in the part of the ball that is located in the upper (moving) die. In contrast, the wall formed in the lower (moving) die is more uniform, and as a result the technological hole has a smaller diameter, with the exception of Case D5 (Fig. 6c). For example, a comparison of Case B5 with Case D5 (or D4) reveals that the formation of a ball from a billet with lower initial wall thickness 
(a)

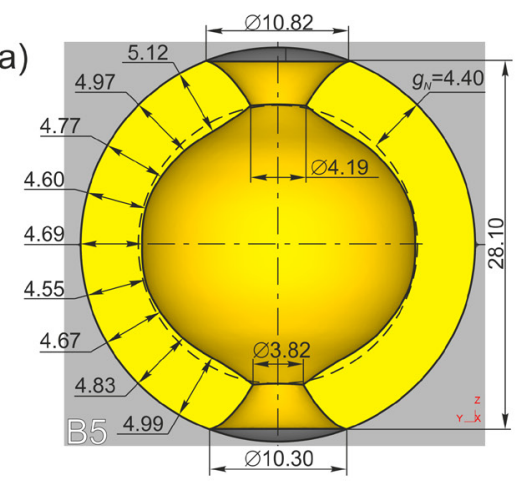

(b)

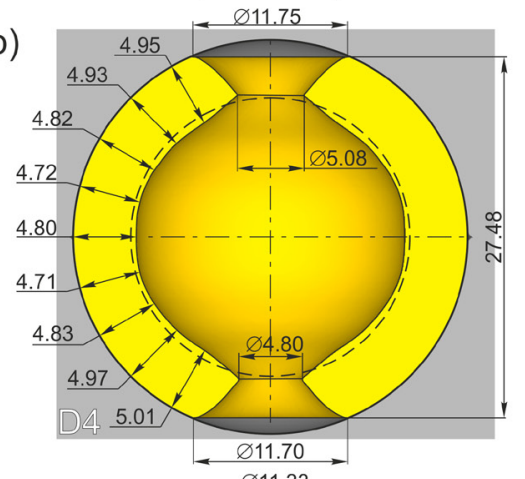

(c)

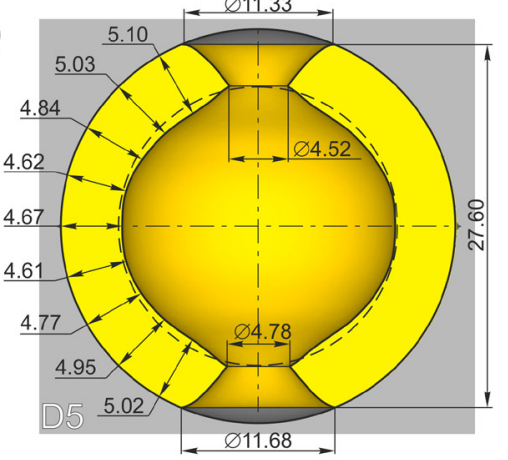

Fig. 6. Cross section of the produced ball with the wall thickness distribution (every $15^{\circ}$ ) and characteristic dimensions, where: a) Case B5, b) Case D4, c) Case D5

$g_{W}$ produces a forging with the wall thickness $g_{K}$ that is closer to the theoretical value $g_{N}$, yet with a higher scatter, while the technological holes have the smallest diameter possible.

Finally, it is necessary to discuss the problem of potential crack occurrence during the forging process, given that the ball is cold formed. To this end, the distribution of the damage function according to the Cockcroft-Latham criterion (C-L function) was determined, as shown in Fig. 7. The highest value of the C-L function was obtained on the external surface of the ball, in the die parting area. This results from the fact that this surface remains free for a long time during the forming process and hence there occur circumferential tensile stresses. However, the highest observed



Fig. 7. Final distribution of the damage function calculated for Case B5

value of the C-L function did not exceed 0.12 , which is considered a safe value for steel. On the other hand, in the other area of the material, the C-L function has relatively low values, which prompts speculation that the workpiece should not undergo cracking during the forging process.

\section{CONCLUSIONS}

The numerical results demonstrate that the proposed cold forging process for producing a hollow steel ball from hollow billet is feasible in real conditions. Based on the obtained results, one can formulate the following general conclusions:

- the forging of hollow ball has a varying wall thickness $g_{K}$, and the formation of two technological holes is inevitable, their diameter $d_{K}$ being a resulting parameter;

- the range of permissible dimensions of a hollow billet is very narrow if one wishes to obtain correctly shaped forgings (i.e. defect-free);

- the use of a billet with the $h_{W} / d_{W}$ ratio exceeding the limit value results in the occurrence of uncontrolled local buckling during the forming process, which - in turn - leads to shape defects such as ball surface concavity and impression underfill;

- if the $h_{W} / d_{W}$ ratio is too low, this requires the use of a billet with the diameter dW that is too big relative to the ball diameter $\mathrm{D}$, which results in the overfill of the working impression of the die and the formation of folds on the external surface of the ball in the vicinity of the die parting plane;

- to obtain the wall thickness distribution that is in line as much as possible with the theo- 
retical value $g_{N}$ and technological holes with the minimal diameter $d_{K}$, it is recommended to use a billet with the smallest wall thickness possible (in the range of permissible billet dimensions); this, however, results in an increase in the $d_{W} / g_{W}$ ratio and a narrow range of the permissible values of other two dimensions, i.e. the diameter $d_{W}$ and the height $h_{W}$.

\section{REFERENCES}

1. Pater Z., Tomczak J. Walcowanie poprzeczno-klinowe kul. Wydaw. Politechniki Lubelskiej, Lublin 2012.

2. Tomczak J., Pater Z., Bulzak T. Designing of screw impressions in the helical rolling of balls. Archives of Civil and Mechanical Engineering, 14, 2014, 104-113.

3. Pater Z. Multi-wedge cross rolling of balls. Journal of Iron and Steel Research International, 20 (10), 2013, 46-50.

4. Pater Z., Tomczak J., Bulzak T. An innovative method for forming balls from scrap rail heads. Advances in Science and Technology Research Journal, 10(31), 2016, 151-157.

5. Kang J.H., Lee H.W. Research on ball forging by ring rolling process. International Journal of Applied Engineering Research, 11 (12), 2016, 7823-7828.

6. Winiarski G. Theoretical analysis of the forging process for producing hallow balls. Advances in
Science and Technology Research Journal, 7 (18), 2013, 68-73.

7. Kwan C.T. A study of process and die design for ball valve forming from stainless steel tube. The International Journal of Advanced Manufacturing Technology, 26 (9-10), 2005, 983-990.

8. Kozjek B., Seruga D., Popelnjak T. Fatigue life prediction of brass ball forging tool. Materials Today: Proceedings, 4 (5), 2017, 5855-5860.

9. Skrochocki D., Tomczaak J. Numerical simulation of rotary compression process of hollow balls. Strength of Materials, 48 (4), 2016, 583-591.

10. Eklund P.E., Campbell G.L. Method of fabricating hollow balls for use in rolling contact bearing applications. US Patent, 3774280A, 1973.

11. Chiuchang S.-Y. Ball valve body manufacturing method. US Patent, 0213125A1, 2003.

12. Alhussainy F., Sheikh M.N., Hadi M.N.S. Behaviour of small diameter steel tubes under axial compression. Structures, 11, 2017, 155-163.

13. Samołyk G. Studies on stress and strain state in cold orbital forging a AlMgSi alloy flange pin. Archives of Metallurgy and Materials, 58 (4), 2013, 1183-1189.

14. Samołyk G. Comparison of the methods for orbital forging illustrated with an example of producing a disk hub. Hutnik. Wiadomości Hutnicze, 81 (9), 2014, 657-662. 